\title{
Factors influencing extracorporeal shock wave lithotripsy efficiency for optimal patient selection
}

\author{
Marius Snicorius, Arnas Bakavicius, Albertas Cekauskas, Marius Miglinas, Gediminas Platkevicius, Arunas Zelvys \\ Institute of Clinical Medicine, Faculty of Medicine, Vilnius University, Vilnius, Lithuania
}

Videosurgery Miniinv 2021; 16 (2): 409-416

DOI: https://doi.org/10.5114/wiitm.2021.103915

\begin{abstract}
Introduction: Current literature suggests various predictors related to the stone and patient, which could influence stone fragmentation and clearance rates.

Aim: To establish clinical characteristics of stone disease for patients undergoing extracorporeal shockwave lithotripsy (ESWL) which may predict the success of the procedure.

Material and methods: One hundred and nine patients with renal stone disease diagnosed by non-contrast computed tomography (NCCT) who underwent ESWL between January 2015 and December 2019 were included in the study. Endpoints: patient being stone free (SF) or when $<4 \mathrm{~mm}$ fragments were detected. Age, gender, location, skinto-stone distance, maximum stone length, stone volume, stone surface area, mean stone Hounsfield units (HU) and highest HU score were explored in uni- and multivariate regression analysis.

Results: Stone size revealed the highest prognostic power for ESWL failure, where OR for stone volume and stone surface area were $1.06(1.03-1.10)$ and $1.04(1.02-1.06)$, respectively (all $p<0.01)$ while a tendency was observed for skin-to-stone distance 1.02 (1.00-1.03). The amount of energy applied during the procedure to one cubic millimeter of stone volume (SMLI/stone volume) was predictive for treatment success (OR =0.60, 95\% CI: 0.41-0.87, $p<$ $0.01)$. Stone volume $(O R=1.06,95 \% \mathrm{Cl}: 1.00-1.14, p=0.01)$ and stone surface area $(O R=1.03,95 \% \mathrm{Cl}: 1.01-1.06$, $p=0.02)$ remained as statistically significant prognostic factors for treatment failure.

Conclusions: Both greater stone volume and stone surface area, as well as lower power delivered per stone volume unit during the ESWL procedure, were found to be significant factors and could be useful to predict treatment failure.
\end{abstract}

Key words: extracorporeal shockwave lithotripsy, kidney stones, stone volume.

\section{Introduction}

In the last decade the prevalence of urolithiasis has increased up to $10.6 \%$ in men and up to $7.1 \%$ in women [1], with especially high recurrence rates $(39 \%$ at 15 years) for both genders [2]. All these changes have led to an increased number of urological procedures for stone disease patients. Thus, as technology has advanced significantly, minimally invasive procedures have gained a huge interest with the intention to reduce the treatment toxicity profile to the minimum.
Extracorporeal shockwave lithotripsy (ESWL) is commonly used to treat patients with upper urinary tract stones. Reports from high volume centers with static machines suggest stone clearance rates of 86-89\%, 71-83\%, 73-84\% and 37-68\% for calculi in the renal pelvis, upper calyx, middle calyx and lower calyx, respectively [3]. Thus, proper patient selection is one of the main cornerstones for improved ESWL efficacy and optimal disease management. Current literature suggests various predictors related to the stone and patient, which could influence stone frag-

\footnotetext{
Address for correspondence

Marius Snicorius, Institute of Clinical Medicine, Faculty of Medicine, Vilnius University, Vilnius, Lithuania,

e-mail: marius.snicorius@gmail.com
} 
mentation and clearance rates $[4,5]$. Such predictors include clinical parameters, such as body mass index as well as computed tomography parameters, such as stone location, skin to stone distance, diameter or stone volume, and Hounsfield units $[5,6]$.

\section{Aim}

The aim of the present study was to establish clinical characteristics of stone disease for patients undergoing ESWL which may contribute to success of the procedure.

\section{Material and methods}

Patients with renal stone disease diagnosed by non-contrast computed tomography (NCCT) who underwent ESWL at a tertiary-level urology center between January 2015 and December 2019 were retrospectively included in the study. Three hundred and five cases were analyzed in total. Of these only 109 patients met study inclusion criteria with accessible NCCT scans and stone size ranging from 5 to $15 \mathrm{~mm}$. The exclusion criteria were absolute contraindications to ESWL such as pregnancy, active urinary infection, uncorrected coagulopathy and patients currently anticoagulated. Patients who did not have NCCT before treatment, maximum stone diameter was over $15 \mathrm{~mm}$ or had multiple stones on the same side were excluded from the study. All patients were treated under the same protocol in the supine position using a Storz Modulith SLK lithotripsy machine using ultrasound guidance (Storz Medical, Germany) without anesthesia. ESWL procedures were performed by four different urologists. During the ESWL procedure up to 3000 shock waves were delivered to the stone with a gradual power increase up to $75 \mathrm{~mJ}$, maintaining frequency of $1.5 \mathrm{~Hz}$ during all the session. The total amount of energy applied to the stone was calculated using the Storz Medical Lithotripsy Index (SMLI). No other treatment, including internal stenting before the procedure or medical expulsion therapy afterwards, was prescribed to the patients.

Variables such as skin-to-stone distance (SSD), maximum stone length (MSL), stone volume (SV), stone surface area (SSA), mean stone density in Hounsfield units (MSD) and highest Hounsfield unit $(\mathrm{HHU})$ score were obtained pre-operatively from NCCT images. SSD was calculated as the average distance from the skin to the surface of targeted stone at $0^{\circ}, 45^{\circ}$ and $90^{\circ}$ angles on NCCT. MSL was measured in the sagittal, transversal and coronal body planes. SV was calculated using the formula: $\mathrm{SV}=l \times w \times d \times \pi \times 0.167$, where $l$ is length, $w$ is width, $d$ is depth and $\pi=3.14159$. For the measurement of stone density, all three body planes were defined for each stone. In each plane an area of interest, smaller than the stone, was depicted where stone density was measured, and the mean value was calculated.

All patients were followed up at the outpatient clinic of the same institution 4 weeks after the treatment. Ultrasonography or NCCT was used to evaluate residual fragments. Success of the procedure was defined as the patient being stone free (SF) or when $<4 \mathrm{~mm}$ fragments were detected. In case of treatment failure, patients underwent repeated ESWL sessions up to 3 times within a 1-month interval between the procedures.

\section{Statistical analysis}

Continuous variables are presented as means with standard deviations (SD). Data for categorical variables are presented as frequencies and percentages. Continuous variables were checked for normal distribution by the Shapiro-Wilk test and compared by the $t$-test when normally distributed or the Mann-Whitney $U$ test for non-normally distributed variables. Pearson's $\chi^{2}$ and Fisher exact tests were used for comparison of categorical variables, as appropriate. To identify predictors for ESWL failure, univariate and multivariate logistic regression analysis was performed, where odds ratios (OR) and $95 \%$ confidence intervals $(\mathrm{Cl})$ were calculated. Receiver operating characteristic (ROC) curves were generated and areas under the curves (AUC) were calculated to compare the predictive power of different characteristics. All statistical tests were performed using SPSS software (IBM Corp., Armonk, NY, USA). P-value of $<0.05$ was considered as statistically significant.

\section{Results}

Overall, 109 patients were included in the study. Baseline demographic and clinical characteristics of the study cohort are summarized in Table I.

Stone size reduction was observed in 77 out of 109 (70.6\%) patients, while success of the procedure was achieved in $73(67.0 \%)$ patients. Treatment failure was associated with larger stones, greater distance to the target and less energy applied to the stone volume unit during the procedure (Table I, all $p<0.05$ ). 
Table I. Baseline demographic and clinical characteristics of the study cohort

\begin{tabular}{|c|c|c|c|c|}
\hline Variable & $\begin{array}{l}\text { All patients } \\
(N=109)\end{array}$ & $\begin{array}{c}\text { Successful } \\
\text { treatment } \\
(N=73)\end{array}$ & $\begin{array}{l}\text { Treatment failure } \\
\qquad(N=36)\end{array}$ & $P$-value* \\
\hline $\begin{array}{l}\text { Age [years] } \\
\text { Mean } \pm \text { SD }\end{array}$ & $54.3(14.7)$ & $55.8(13.8)$ & $51.2(16.7)$ & 0.13 \\
\hline \multicolumn{5}{|l|}{ Gender, $n(\%)$ : } \\
\hline Female & $46(42.2)$ & $32(43.8)$ & $14(38.9)$ & 0.68 \\
\hline Male & $63(57.8)$ & $41(56.2)$ & $22(61.1)$ & \\
\hline \multicolumn{5}{|l|}{ Stone location, $n(\%)$ : } \\
\hline Lower calyx & $54(49.5)$ & $37(50.7)$ & $17(47.2)$ & 0.83 \\
\hline Other & $55(50.5)$ & $36(49.3)$ & $19(52.8)$ & \\
\hline $\begin{array}{l}\text { Max. stone diameter }[\mathrm{mm}] \\
\text { Mean }( \pm \mathrm{SD})\end{array}$ & $10.0(3.6)$ & $8.3(2.6)$ & $10.8(3.7)$ & 0.01 \\
\hline $\begin{array}{l}\text { Stone volume }\left[\mathrm{mm}^{3}\right] \\
\text { Mean }( \pm \mathrm{SD})\end{array}$ & $269.5(232.7)$ & $150.8(123.3)$ & $328.0(251.6)$ & 0.01 \\
\hline $\begin{array}{l}\text { Stone surface area }\left[\mathrm{mm}^{2}\right] \\
\text { Mean }( \pm \mathrm{SD})\end{array}$ & $54.3(35,1)$ & $36.8(23.0)$ & $62.8(36.9)$ & 0.02 \\
\hline \multicolumn{5}{|l|}{ Stone attenuation value [HU]: } \\
\hline Maximum ( \pm SD) & $1170.9(375.6)$ & $1092.4(422.6)$ & 1209.7 (346.6) & 0.12 \\
\hline Mean stone density $( \pm \mathrm{SD})$ & $804.2(282.1)$ & $762.1(301.0)$ & $824.9(271.9)$ & 0.27 \\
\hline $\begin{array}{l}\text { Skin to stone distance }[\mathrm{mm}] \\
\text { Mean }( \pm \mathrm{SD})\end{array}$ & $110.2(24.3)$ & $104.4(20.8)$ & $113.0(25.5)$ & 0.03 \\
\hline $\begin{array}{l}\text { SMLI } \\
\text { Mean }( \pm \text { SD) }\end{array}$ & $167.3(33.1)$ & $167.4(32.4)$ & $167.3(35.1)$ & 0.98 \\
\hline $\begin{array}{l}\text { SMLI/stone volume ratio } \\
\text { Mean }( \pm \text { SD) }\end{array}$ & $1.3(1.5)$ & $2.0(1.8)$ & $1.0(1.1)$ & 0.01 \\
\hline
\end{tabular}

HU - Hounsfield units, $N$ - number of patients, SD - standard deviation, SMLI - Storz Medical Lithotripsy Index. ${ }^{*}$-values calculated for comparison of successful and failed treatment cohorts only. Statistically significant $p$-values are marked in bold.

During univariate logistic regression analysis stone size outperformed other clinical characteristics and revealed the highest prognostic power for ESWL failure, where OR for stone volume and stone surface area were $1.06(1.03-1.10)$ and 1.04 $(1.02-1.06)$, respectively (Table II, all $p<0.01$ ) while a tendency was observed for skin to stone distance 1.02 (1.00-1.03). Amount of energy applied during the procedure to one cubic millimeter of stone volume (SMLI/stone volume) was predictive for treatment success (OR $=0.60,95 \% \mathrm{Cl}: 0.41-0.87, p<$ 0.01). In multivariate logistic regression analysis (Table II), stone volume $(\mathrm{OR}=1.06,95 \% \mathrm{Cl}: 1.00-1.14$, $p=0.01)$ and stone surface area $(\mathrm{OR}=1.03,95 \% \mathrm{Cl}$ : $1.01-1.06, p=0.02$ ) remained as statistically significant prognostic factors for treatment failure.

In ROC analysis (Figure 1), stone volume was the most significant independent predictor for ESWL failure, where AUC for stone volume was 0.754 $(p<0.01)$ and AUC for stone surface area was 0.742 $(p<0.01)$, while the same tendency was observed for skin to stone distance (AUC $=0.594, p=0.10$ ).

\section{Discussion}

The ESWL procedure provides good treatment outcomes [6] with similar overall complication rates as for endourological procedures, such as retrograde ureteroscopy [6, 7]. However, many factors, associated with stone characteristics and constitution of the patient, as well as technical aspects of ESWL, have been identified to influence treatment efficacy $[4,5]$.

According to the literature, stone-free status after ESWL monotherapy for stones of size $<20 \mathrm{~mm}$ is $80-85 \%[8,9]$, as compared to $33-65 \%$ for stones $>20 \mathrm{~mm}[5,10]$. Taking all these findings into consideration, ESWL is recommended for kidney calculi smaller than $20 \mathrm{~mm}$ [11]. These findings are in line 
Table II. Univariate and multivariate logistic regression analysis of the associations between clinical characteristics and treatment failure

\begin{tabular}{|lccccccc|}
\hline Variable & \multicolumn{3}{c}{ Univariate } & \multicolumn{3}{c|}{ Multivariate } \\
\cline { 2 - 8 } & OR & $95 \% \mathrm{Cl}$ & $P$-value & OR & $95 \% \mathrm{Cl}$ & $P$-value \\
\hline Stone volume & 1.06 & $1.03-1.10$ & $\mathbf{0 . 0 1}$ & 1.06 & $1.00-1.10$ & $\mathbf{0 . 0 1}$ \\
\hline Stone surface area & 1.04 & $1.02-1.06$ & $\mathbf{0 . 0 1}$ & 1.03 & $1.01-1.06$ & $\mathbf{0 . 0 2}$ & 0.73 \\
\hline Mean stone density & 1.00 & $0.99-1.00$ & 0.28 & 1.00 & $0.99-1.02$ & 0.36 \\
\hline Skin to stone distance & 1.02 & $1.00-1.03$ & 0.09 & 1.01 & $0.99-1.03$ & $0.60-1.38$ & 0.64 \\
\hline SMLI/stone volume ratio & 0.60 & $0.41-0.87$ & $<0.01$ & 0.91 & 0.63 & & \\
\hline
\end{tabular}

$\mathrm{Cl}$ - confidence interval, $\mathrm{OR}$ - odds ratio. Statistically significant $p$-values are marked in bold.

with our results, where stone size was significantly associated with treatment outcomes and greater stone volume was detected in patients with ESWL failure.

Lower pole renal calculi provide a unique challenge when considering the ESWL procedure due to a lower stone clearance rate - 52-69\% [12-14]. In

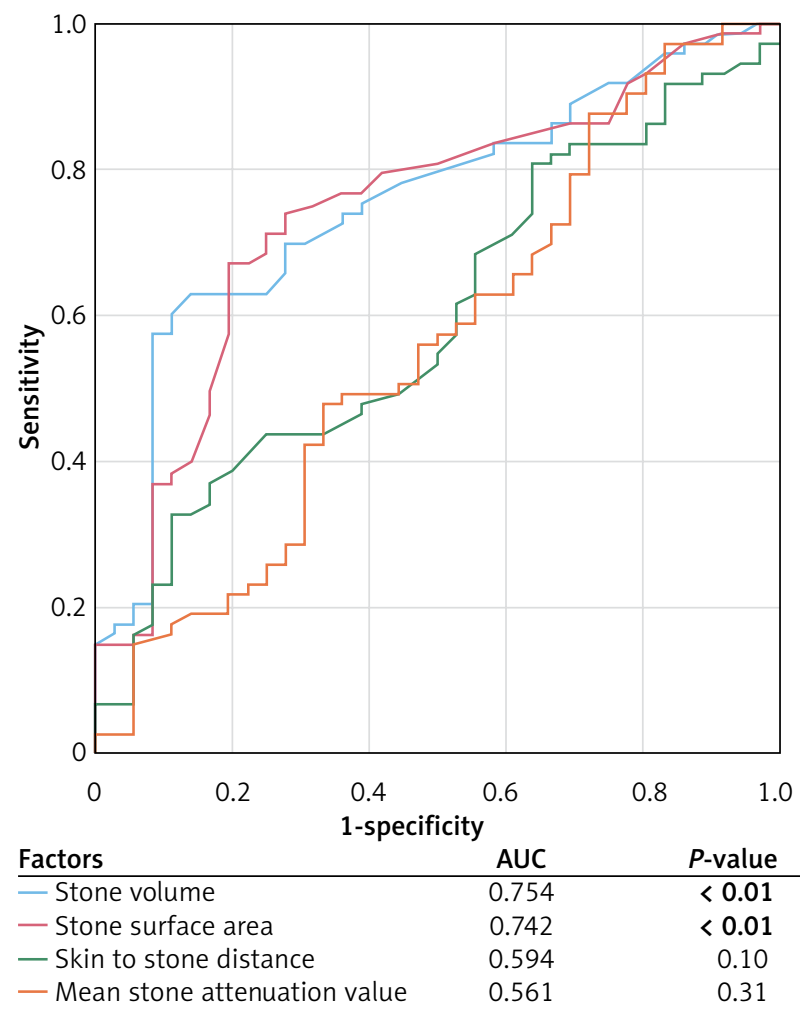

Figure 1. Factors influencing extracorporeal shock wave lithotripsy efficiency for optimal patient selection. ROC analysis results for ESWL failure

AUC - area under the curve; statistically significant $p$-values are marked in bold. our study a considerably high number of patients (49.5\%) underwent ESWL due to calculi in the inferior calyx, while no significant differences were observed in primary treatment outcomes. All these findings confirm the idea that stone disintegration efficacy of the ESWL procedure is the same in any part of the kidney, while stone clearance from the lower part of the kidney could be affected by the infundibular angle or a narrow calyx.

Increased BMI could be an independent predictor for ESWL outcomes. Pareek et al. evaluated patients who underwent ESWL for a solitary renal stone measuring between 5 and $10 \mathrm{~mm}$ using an electrohydraulic lithotripter. After follow-up $72 \%$ were stone free and $28 \%$ had residual fragments larger than $3 \mathrm{~mm}$. The stone-free patients had a significantly smaller mean BMI $\left(26.9 \pm 0.5 \mathrm{~kg} / \mathrm{m}^{2}\right)$ than patients with residual fragments $(30.8 \pm 0.9)$. Logistic regression analysis performed by the authors revealed that an unsuccessful outcome was statistically significantly related to the $\mathrm{BMI}(\mathrm{OR}=1.34, p<0.01)[15]$. In a larger cohort of 688 patients, Delakas et al. using a second-generation electrohydraulic shockwave lithotripter found no association between increased BMI and ESWL failure [16]. However, more studies suggest that high BMI affects outcomes of the ESWL. Garrido-Abdad et al. also reported that high BMI was one of the parameters that showed a significant difference after multivariate analysis. The cut-off value was $26.9 \mathrm{~kg} / \mathrm{m}^{2}$ [17].

Skin-to-stone distance (SSD) is mostly calculated as the mean of the distances from the body surface to a targeted stone at $0^{\circ}, 45^{\circ}$, and $90^{\circ}$ angles on NCCT using radiographic calipers. BMI is an important parameter in predicting ESWL success. Some authors have concluded that the SSD value is a more predic- 
tive parameter than BMI due to different body types and body fat distribution among people and races. Pareek et al. revealed that SSD (using a cutoff value of $10 \mathrm{~mm}$ ) was a much more powerful predictor than $\mathrm{BMI}$ and hypothesized that the travelling of shock waves for longer distances is associated with attenuation of those shock waves [4]. Similarly, Wiesenthal et al. reported that the cutoff value for SSD was $11 \mathrm{~mm}$ [18]. It has been noted in previous studies that failure of ESWL is related to greater SSD. The mean SSD for ESWL success was $8.12 \pm 1.74 \mathrm{~cm}$ against $11.53 \pm 1.89 \mathrm{~cm}$ in the ESWL failure group ( $p<0.01$ ) [15]. According to another study, the mean SSD in the stone-free group was $83.3 \pm 21.9 \mathrm{~mm}$ compared to the residual stone group where the mean SSD was $107.7 \pm 28.9 \mathrm{~mm}(p<0.050)$ and SSD was the only significant independent predictor of treatment outcome [19]. A success rate of $71.4 \%$ was noted in patients having SSD less than $100 \mathrm{~mm}$ in contrast to $46.2 \%$ in those with SSD greater than $100 \mathrm{~mm}$, showing the OR of 1.036 (95\% Cl: $1.014-1.059$; $p<0.01)$. Moreover, SSD was noted to be $90.65 \mathrm{~mm}$ in the success group compared with $104.33 \mathrm{~mm}$ in the failure group, which favors the suggested cut-off value [13]. Shinde et al. reported that in their study the success group had a mean SSD of $103.9 \pm 21.3$ $\mathrm{mm}$ while the mean SSD in the failure group was $111.6 \pm 22.4 \mathrm{~mm}$ [20]. Our analysis results are in line with other authors, where the successful treatment group had a mean SSD of $104.4 \pm 20.8 \mathrm{~mm}$ and the failure group $113.0 \pm 25.5 \mathrm{~mm}(p<0.01)$.

However, there are some studies in Asian populations with contradictory results. Because Asian populations have thin bodies compared to Western populations, it was argued that it could not be applied to Asian patients. Various studies reported that SSD was not a meaningful factor [21].

Stone attenuation value (SAV) is mostly measured by creating three regions of interest in three different views of the stone on the NCCT scan showing the stone in the largest dimension. It is presented by the mean values of defined stone regions in Hounsfield unit (HU) [13]. Other authors calculated SAV using mean attenuation of three consistent (area $0.02 \mathrm{~cm}^{2}$ ), non-overlapping regions of interest chosen from stones in bone windows [21].

There is a conclusion that stones with higher density require a larger amount of shock waves [15, 21, 22]. Furthermore, in 2013, Hameed et al. [23] reported similar results and concluded that stones having > 1,350 $\mathrm{HU}$ require increased shock wave energy.

Nowadays most authors use a 815-1000 HU cut-off value for predicting successful ESWL. Shinde et al. observed a stone-free rate of $56.2 \%$ in patients with stones $>1000 \mathrm{HU}$ density compared to that of $87.7 \%$ with a stone density $\leq 1000 \mathrm{HU}$ [20]. In Wiesenthal's study and another by Wang, stones with $>900 \mathrm{HU}$ were more likely to fail after $\operatorname{ESWL}[18,24]$. Nakasato et al. reported that success rates following ESWL were significantly higher for stones $<815 \mathrm{HU}$ than for stones > $815 \mathrm{HU}(p<0.02)$ [25]. In another study, Quzaid et al. reported that stone density of $970 \mathrm{HU}$ was the most sensitive point in the determination of stone density. They achieved $96 \%$ stonefree rates for stones $<970 \mathrm{HU}$ and 38\% stone-free rates for stones $\geq 970 \mathrm{HU}(p<0.001)$ [26].

Patients having SAV less than $500 \mathrm{HU}$ were much more likely to have stone clearance (93.8\%), while those having values greater than 1,000 $\mathrm{HU}$ were much less likely to experience successful outcomes $(24.5 \%)$, even with an increasing number of shock waves [13]. Other authors also published perfect results for patients with stones < $500 \mathrm{HU}$ [14]. In our study mean stone density was not significantly different between successful ESWL and failure groups, but patients with mean stone density lower than $500 \mathrm{HU}$ had successful treatment outcome in $71.1 \%$ of cases while patients with higher than $500 \mathrm{HU}$ had a positive outcome only in $47.4 \%$ of cases $(p=0.06)$.

With the frequent use of NCCT to evaluate stone disease, stone burden is commonly measured using the axial stone diameter. The size of the stone is typically assessed by measuring the maximum length. However, kidney stones are irregular 3D structures and can have complex geometric shapes. The stone surface area is a 2D measurement of stone burden, as it takes into account the overall shape of the stone. Both stone length and surface area measured by traditional radiography fail to provide any volumetric information, as they are limited by the inability to measure the third dimension (depth of the stone). Importantly, the shape and depth of the stone might have significant implications for the total stone burden. The total volume of a cylinder is twice the volume of a sphere of the same diameter and height. These differences might be even more important when measuring the stone burden in patients with irregularly shaped stones [27]. 
Bandi et al. analyzed how stone volume predicts outcomes of ESWL. The mean stone volume was significantly different between stone-free and residual fragment groups (274 vs. $464 \mu \mathrm{l}$ ) and the stone volume was the strongest predictor of stone-free status. A stone volume of $<500 \mu \mathrm{l}$ best predicted treatment success with $72 \%$ of patients with a stone volume of $<500 \mu$ l having a successful outcome vs. only $27 \%$ with a stone volume of $>500 \mu \mathrm{l}$ [27]. Similarly, El-Nahas claimed SV as a predictive factor for disintegration of stones following ESWL [28].

Our successfully treated patient group had a mean stone volume of $150.8 \pm 123.3 \mathrm{~mm}^{3}$ and the failure group $328.0 \pm 251.6 \mathrm{~mm}^{3}$ ( $p<0.01$ ). During univariate logistic regression analysis stone size showed the highest prognostic power for ESWL failure, where ORs for stone volume and stone surface area were 1.06 (1.03-1.10) and 1.04 (1.02-1.06). In multivariate logistic regression analysis including all significant covariates, both stone volume $(O R=1.06$, 95\% Cl: $1.00-1.14, p=0.01)$ and stone surface area $(\mathrm{OR}=1.03,95 \% \mathrm{Cl}: 1.01-1.06, p=0.02)$ were shown as prognostic factors for treatment failure.

A study of Tran et al. presented a simple metric for stone volume and called it ellipsoid stone volume (ESV). This metric was developed for the purpose of overcoming difficulties calculating stone volume. ESV can be rapidly determined with measurement of the anteroposterior, horizontal, and craniocaudal stone diameters. Notably, when ESV is compared with the computer-generated 3D stone volume, the correlation coefficient is 0.9893 . The authors found that ESV is a strong predictor of ESWL success, with an AUC of 0.775 [29]. Waqas et al. noted a correlation between the stone burden (in terms of stone size or diameter, stone area, and stone volume) and the success rate. Stone volume $\left(\mathrm{mm}^{3}\right)$ in the success group was $515.44 \pm 628.05$ and $1,118.31 \pm 1,335.74$ in the failure group. However, this again failed to show any significance in the multivariate analysis. This may be attributable to the homogeneity of stone size within their study population resulting in a type I error [13].

Application of optimal shock wave rates can both improve stone fragmentation and reduce surrounding tissue damage [30]. Kang et al. in a systematic review and network meta-analysis compared low $(1 \mathrm{~Hz})$, intermediate $(1.5 \mathrm{~Hz})$ and high $(2 \mathrm{~Hz})$ lithotripsy rates. Thirteen RCTs were included, showing that success rates of low $(O R=2.2)$ or intermedi- ate frequency ESWL $(O R=2.5)$ were greater than for high-frequency ESWL. There was no significant difference in ESWL success rate for low and intermediate frequency ESWL [31].

A study from Hong Kong assessed the effects of a ramping protocol in patients undergoing extracorporeal shock wave lithotripsy of renal stones. They randomized patients into two groups: group 1 (first 1,000 shocks at energy level 5 followed by 1,000 shocks at energy level 6 and 1,000 final shocks at energy level 7) and a fixed voltage protocol in group 2 (all 3,000 shocks at energy level 7). Group 1 received $14.8 \%$ lower energy than group 2, which was significant $(p<0.001)$. The treatment success rate in groups 1 and 2 was $67.8 \%$ and $73.6 \%$, respectively, which was not significant (group 1 crude OR $=0.753,95 \% \mathrm{Cl}: 0.456-1.244, p=0.268)$. The difference in stone-free rates in groups 1 and 2 was $36.6 \%$ and $41.9 \%$ and was not significant as well. However, there was a significant difference in perinephric hematoma development rates in group 1 and 2 , observed in $23.8 \%$ and $43.8 \%$ of patients respectively $(p<0.001)$ [32]. In our study we used 90 shocks per minute during ESWL procedures. Power ramping also was used. Shock wave power was gradually increased up to $75 \mathrm{~mJ}$. We did not find any current studies analyzing how power delivered per stone volume unit affects ESWL efficiency. During our analysis, power delivered to the stone was calculated using the SMLI index (shock waves power adjusted by shock waves rate). Successfully treated patients had a mean SMLI/stone volume ratio of $2.0 \pm 1.8$ while the failure group had a lower ratio of $1.0 \pm 1.1(p=0.01)$. These results show that power delivered for a single unit of stone volume is an important factor for ESWL outcome. SMLI/stone volume was a predictive factor for treatment success $(\mathrm{OR}=0.60,95 \% \mathrm{Cl}: 0.41-0.87, p<0.01)$. These findings definitely require further investigation and the optimal cut-off value is still unknown.

The impact of patient's age on ESWL outcomes is debatable. Many studies have discussed factors affecting the outcome of ESWL, but only a few have considered age of any significance. One study of 3023 patients with renal and ureteric calculi treated with ESWL revealed that a significantly lower stonefree rate was associated with older age [5]. Another multivariate analysis of 2954 patients with kidney stones treated with ESWL revealed that patients aged $>40$ years had a significantly poorer stone-free 
rate [33]. The reason for this finding is still unknown. Age-related sclerotic kidney changes may affect the acoustic impedance and lower efficacy of ESWL. Another factor could be reduced physical activity. Further studies are needed to analyze age as a predictor for ESWL outcome.

Although still under investigation, clinical nomograms have been used to guide physicians in selecting the safest and most effective treatment for specific patients. Some authors have demonstrated that when information from nomograms was used to select patients to undergo ESWL, success rates were higher [34]. Although in practice these nomograms may be complex and confusing [4, 5], recently Tran et al. reported a novel and simple nomogram, called the "Triple D scoring system". Using computed tomography imaging, the Triple D describes three parameters: stone density, stone volume (SV), and skin-to-stone distance (SSD). The authors concluded that this scoring system may increase the success rates of ESWL by indicating appropriate patients for the treatment. The Triple D score integrates three powerful predictors of ESWL success into a single score, where a score of $0,1,2$, and 3 correlates with success rates of $21.4 \%, 41.3 \%, 78.7 \%$, and $96.1 \%$, respectively [34]. Some authors criticize this system due to a lack of external validation and short postoperative follow-up period, which may underestimate the stone-free rates [33]. Ozgor et al. completed a study to externally validate the Triple D score. The conclusion was that Triple D scores were significantly higher in patients successfully treated with ESWL compared with patients in whom ESWL failed $(p<0.001)$. Triple D scores of $0,1,2$, and 3 correlated with stone-free rates of $41.7 \%, 33.7 \%, 69.4 \%$, and $97 \%$, respectively. The multivariate analyses revealed that Triple D score and stone location were independent factors affecting ESWL success [33].

We understand that we are prevented by the sample size and retrospective nature of the study from drawing strong conclusions. Moreover, according to our practice we have evaluated outcomes 4 weeks after the procedure. Therefore, we could expect an additional fragment passing during the period of 3 months. This could lead to an overestimation of our failure rates. However, our data could be used to identify the patients who could have an early benefit from ESWL treatment in order to give the objective information to the patient before choosing an appropriate personalized treatment method.
From our point of view predictive factors taken separately cannot identify all patients who are likely to benefit from ESWL and exclude those that will have an unfavorable outcome. A modern approach should be used combining various factors, including stone location, size, skin-stone distance, BMI, and stone density. Delivered power and stone volume ratio could be useful tools to calculate the required power to fragment the stone. Specialized and verified nomograms may help to improve patient selection for ESWL procedures and further investigation is needed.

\section{Conclusions}

Both greater stone volume and stone surface area, as well as lower power delivered to the stone volume unit during the ESWL procedure could be useful to predict treatment failure. The most important predictive factor of treatment failure is stone volume. In our opinion, power delivered to the stone (SMLI) and stone volume ratio could be useful tools for ESWL procedure planning. This information can facilitate better procedure planning and patient information. Further larger prospective studies are required to verify our results.

\section{Research involving human participants}

The authors certify that the study was performed under the ethical standards as laid down in the 1964 Declaration of Helsinki and its later amendments or comparable ethical standards.

\section{Conflict of interest}

The authors declare no conflict of interest.

\section{References}

1. Knoll T, Pearle MS. Clinical management of urolithiasis. Springer Verlag, Berlin, Heidelberg 2013.

2. Rule AD, Lieske JC, Li X, et al. The ROKS nomogram for predicting a second symptomatic stone episode. J Am Soc Nephrol 2014; 25: 2878-86.

3. Nielsen TK, Jensen JB. Efficacy of commercialised extracorporeal shock wave lithotripsy service: a review of 589 renal stones. BMC Urol 2017; 17: 59.

4. Pareek G, Hedican SP, Lee FT, et al. Shock wave lithotripsy success determined by skin-to-stone distance on computed tomography. Urology 2005; 66: 941-4.

5. Abe T, Akakura K, Kawaguchi M, et al. Outcomes of shockwave lithotripsy for upper urinary-tract stones: a large-scale study at a single institution. J Endourol 2005; 19: 768-73. 
6. Fankhauser CD, Hermanns T, Lieger L, et al. Extracorporeal shock wave lithotripsy versus flexible ureterorenoscopy in the treatment of untreated renal calculi [published correction appears in Clin Kidney J 2018; 12: 309-10]. Clin Kidney J 2018; 11: 364-9.

7. Iqbal N, Malik Y, Nadeem U, et al. Comparison of ureteroscopic pneumatic lithotripsy and extracorporeal shock wave lithotripsy for the management of proximal ureteral stones: a single center experience. Turk J Urol 2018; 44: 221-7.

8. Miller NL, Lingeman JE. Management of kidney stones. BM 2007; 334: 468-72

9. Khalil MM. Which is more important in predicting the outcome of extracorporeal shockwave lithotripsy of solitary renal stones: stone location or stone burden? J Endourol 2012; 26: 535-9.

10. Lingeman JE, Lifshitz DA, Evan AP. Surgical management of urinary lithiasis. In: Campbell's Urology. Walsh PC (ed.). $8^{\text {th }}$ ed. WB Saunders, Philadelphia 2002; 3361-451.

11. Türk C (Chair), Neisius A, Petrik A, Seitz C, Skolarikos A (Vicechair), Thomas K. EAU Guidelines. Edn. presented at the EAU Annual Congress Amsterdam 2020. ISBN 978-94-92671-07-3.

12. Davis NF, Donaldson JF, Lombardo R, et al. Frequency and factors effecting non clearance of lower pole renal stones. J Ayub Med Coll Abbottabad 2015; 27: 384-7.

13. Waqas M, Saqib IU, Imran Jamil M, et al. Evaluating the importance of different computed tomography scan-based factors in predicting the outcome of extracorporeal shock wave lithotripsy for renal stones. Investig Clin Urol 2018; 59: 25-31.

14. Massoud AM, Abdelbary AM, Al-Dessoukey AA, et al. The success of extracorporeal shock-wave lithotripsy based on the stone-attenuation value from non-contrast computer tomography. Arab J Urol 2014; 12: 155-61.

15. Pareek G, Armenakas NA, Panagopoulos G, et al. Extracorporeal shock wave lithotripsy success based on body mass index and Hounsfield units. Urology 2005; 65: 33-6.

16. Delakas D, Karyotis I, Daskalopoulos G, et al. Independent predictors of failure of shockwave lithotripsy for ureteral stones employing a second-generation lithotripter. J Endourol 2003; 17: 201-5.

17. Garrido-Abad P, Rodríguez-Cabello MÁ, Platas-Sancho A. Analysis of success predictive factors in the treatment of urinary lithiasis by extracorporeal shock wave lithotripsy. patient optimization: ESWL score. Arch Esp Urol 2017; 70: 715-24.

18. Wiesenthal JD, Ghiculete D, Ray AA, et al. A clinical nomogram to predict the successful shock wave lithotripsy of renal and ureteral calculi. J Urol 2011; 186: 556-62.

19. Patel T, Kozakowski K, Hruby G, et al. Skin to stone distance is an independent predictor of stone-free status following shockwave lithotripsy. J Endourol 2009; 23: 1383-5.

20. Shinde S, Al Balushi Y, Hossny M, et al. Factors affecting the outcome of extracorporeal shockwave lithotripsy in urinary stone treatment. Oman Med J 2018; 33: 209-17.

21. Cho KS, Jung HD, Ham WS, et al. Optimal skin-to-stone dis tance is a positive predictor for successful outcomes in upper ureter calculi following extracorporeal shock wave lithotripsy: a bayesian model averaging approach. PLoS One 2015; 10 e0144912.
22. Gupta NP, Ansari MS, Kesarvani P, et al. Role of computed tomography with no contrast medium enhancement in predicting the outcome of extracorporeal shock wave lithotripsy for urinary calculi. BJU Int 2005; 95: 1285-8.

23. Hameed DA, Elgammal MA, ElGanainy EO, et al. Comparing non contrast computerized tomography criteria versus dual X-ray absorptiometry as predictors of radio-opaque upper urinary tract stone fragmentation after electromagnetic shockwave lithotripsy. Urolithiasis 2013; 41: 511-5.

24. Wang LJ, Wong YC, Chuang CK, et al. Predictions of outcomes of renal stones after extracorporeal shock wave lithotripsy from stone characteristics determined by unenhanced helical computed tomography: a multivariate analysis. Eur Radiol 2005; 15 : 2238-43.

25. Nakasato T, Morita J, Ogawa Y. Evaluation of Hounsfield units as a predictive factor for the outcome of extracorporeal shock wave lithotripsyand stone composition. Urolithiasis 2015; 43: 69-75.

26. Ouzaid I, Al-qahtani S, Dominique S, et al. A 970 Hounsfield units $(\mathrm{HU})$ threshold of kidney stone density on non-contrast computed tomography (NCCT) improves patients' selection for extracorporeal shockwave lithotripsy (ESWL): evidence from a prospective study. BJU Int 2012; 110: E438-42.

27. Bandi G, Meiners RJ, Pickhardt PJ, et al. Stone measurement by volumetric three-dimensional computed tomography for predicting the outcome after extracorporeal shock wave lithotripsy. BJU Int 2009; 103: 524-8.

28. El-Nahas AR, El-Assmy AM, Mansour O, et al. A prospective multivariate analysis of factors predicting stone disintegration by extracorporeal shock wave lithotipsy: the value of high-resolution non contrast computed tomography. Eur Urol 2007; 51: 1688-93.

29. Tran TY, McGillen K, Blanchard Cone E, et al. Triple D Score is a reportable predictor of shockwave lithotripsy stone-free rates. J Endourol 2015; 29: 226-30.

30. Anglada-Curado FJ, Campos-Hernandez P, Carrasco-Valiente J, et al. Extracorporeal shock wave lithotripsy for distal ureteral calculi: improved efficacy using low frequency. Int I Urol 2013; 20: 214-9.

31. Kang DH, Cho KS, Ham WS, et al. Comparison of high, intermediate, and low frequency shock wave lithotripsy for urinary tract stone disease: systematic review and network meta-analysis. PLoS One 2016; 11: e0158661.

32. Ng CF, Yee CH, Teoh JYC, et al. Effect of stepwise voltage escalation on treatment outcomes following extracorporeal shock wave lithotripsy of renal calculi: a prospective randomized study. J Urol 2019; 202: 986-93.

33. Abdel-Khalek M, Sheir KZ, Mokhtar AA, et al. Prediction of success rate after extracorporeal shock-wave lithotripsy of renal stones - a multivariate analysis model. Scand J Urol Nephrol 2004; 38: 161-7.

34. Ozgor F, Tosun M, Kayali Y, et al. External validation and evaluation of reliability and validity of the triple $d$ score to predict stone-free status after extracorporeal shockwave lithotripsy. J Endourol 2017; 31: 169-73.

Received: 30.09.2020, accepted: 6.11.2020 